\title{
An extended life time distribution: theory, properties and applications
}

\author{
Salman Abbas*(iD, Muhammad Mohsin(D) \\ Department of Statistics, COMSATS University Islamabad, Lahore Campus, Lahore Pakistan
}

\begin{abstract}
This paper is an addition to the series of modification and improvement of the extant distributions which enable them to analyze the new emerging situations efficiently. We develop a new lifetime distribution by generalizing the Erlang truncated exponential distribution using the Topp Leone family of distributions. A comprehensive account of mathematical characteristics such as quantile function, moments, probability weighted moments, moment generating function, and probability generating function of the proposed distribution is presented. Some reliability measures such as hazard rate function, residual life function, and reversed residual life function are also provided. Several entropy measures including Rèyni entropy, Tsallis entropy, cumulative Tsallis entropy, and dynamic cumulative Tsallis entropy are obtained. Besides, the extropy, residual extropy, and cumulative residual extropy are explored. The unknown parameters of the proposed distribution are estimated by using the maximum likelihood method. The stability of the model parameters is examined through the simulation study. The application of our proposed distribution is explained through three real-life examples and its performance is illustrated through its comparison with the competent existing distributions.
\end{abstract}

Mathematics Subject Classification (2020). 60E06

Keywords. Erlang truncated exponential distribution, Topp Leone family of distributions, distributional characteristics, entropy, reliability measures, inferences

\section{Introduction}

Probability distributions comprehensively analyze different emerging situations and provide possible solutions leading toward important inferences. Besides the development of new distributions, the generalization of the classical distributions has been a very attractive practice. The resulting generalized distributions are applied in the modeling of real-life data sets effectively in almost all the disciplines such as biology, environmental sciences, survival analysis, medical science, engineering, reliability analysis etc.

The exponential model is widely used in reliability engineering because it has simple expression and analytical tractability. It is one of the well known classical distributions used for generalization due to its interesting "lack of memory property". Many generalizations

\footnotetext{
*Corresponding Author.

Email addresses: salmanabbas@cuilahore.edu.pk (S. Abbas), muhammadmohsin@cuilahore.edu.pk (M. Mohsin)

Received: 28.08.2020; Accepted: 26.07.2021
} 
of the exponential distribution are available in the literature. Some prominent generalized distributions are mentioned as; an extended exponentiated exponential distribution by [2], the extended exponential distribution by [3], generalized exponential distributions by [9], the beta exponential distribution by [17], and moment distribution by [6].

El-Alosey [7] introduces a new two parameters exponential distribution named as Erlang truncated exponential (ETEx) distribution. The distribution function (cdf) of the ETEx distribution is given as

$$
H(y)=1-e^{-\alpha\left(1-e^{-\gamma}\right) y}, \quad y>0, \alpha, \gamma>0,
$$

the corresponding density function (pdf) is obtained by differentiating (1.1) and given as

$$
h(y)=\alpha\left(1-e^{-\gamma}\right) e^{-\alpha\left(1-e^{-\gamma}\right) y}, \quad y>0, \alpha, \gamma>0,
$$

where $\alpha$ is the shape parameter and $\gamma$ is the scale parameter.

Many generalizations of ETEx distribution exist in the literature. Okorie et al. [20] define Transmuted Erlang truncated exponential distribution, Nasiru et al. [18] present generalized Erlang truncated exponential distribution, Okorie et al. [22] develop MarshallOlkin generalized Erlang truncated exponential distribution, Okorie [21] derives the extended Erlang truncated exponential distribution, Nasiru et al. [19] introduce Poisson exponentiated Erlang truncated exponential distribution and Mohsin et al. [14] study the characterization of the Erlang truncated exponential distribution.

Nadarajah and Kotz [16] propose Topp Leone distribution which is taken as a replacement of Beta distribution. The cdf of Topp Leone distribution is in closed form which not only increases its scope but also makes it analytically pliable. Al-Shomrani et al. [4] construct a generalized family of Topp Leone distribution having the following cdf

$$
F_{T L-H}(y)=[H(y)]^{b}[2-H(y)]^{b}=\left[1-(\bar{H}(y))^{2}\right]^{b}, \quad x \epsilon \Re, b>0,
$$

with the corresponding pdf as

$$
f_{T L-h}(y)=2 b g(t) \bar{H}(y)\left[1-(\bar{H}(y))^{2}\right]^{b-1}, b>0,
$$

where $g(y)=H^{\prime}(y)$ and $\bar{H}(y)=1-H(y)$.

Since ETEx distribution has a constant failure rate, therefore, it cannot model the intricate situations having a non-constant failure rate as given by [22]. This paper aims to provide a new lifetime distribution by generalizing the ETEx distribution using Equation (1.3). The resulting generalized distribution is named as Topp Leone Erlang truncated exponential (TL-ETEx) distribution. This distribution is capable of modeling the situations having either constant failure rate or non-constant failure rate. Moreover, we present three real-life examples from hydrology, reliability and environmental sciences which support the better fitting of the proposed distribution. We hope this generalization might help to model the complex situations in real life.

The article is unfolded as: In Section 2, the model is developed and its characteristics are derived. In Section 3, measures of entropy and extropy are explored. In Section 4, model parameters are estimated along with the Fisher information matrix. In Section 5 , a simulation study is performed. In Section 6, applicability of the proposed model is establisheded in three different fields. In Section 7, some concluding remarks are stated.

\section{TL-ETEx distribution and its characteristics}

In this section, the expressions for the cdf and the pdf of the Topp Leone Erlang truncated exponential (TL-ETEx) distribution are derived along with several of its characteristics and reliability measures. 
The cdf of the TL-ETEx distribution is obtained by inserting Equation (1.3) in Equation (1.1) as

$$
F(y)=\left[1-e^{-2 \alpha\left(1-e^{-\gamma}\right) y}\right]^{b},
$$

and the corresponding pdf is derived by differentiating Equation (2.1) as

$$
f(y)=2 b \alpha\left(1-e^{-\gamma}\right) e^{-2 \alpha\left(1-e^{-\gamma}\right) y}\left[1-e^{-2 \alpha\left(1-e^{-\gamma}\right) y}\right]^{b-1}, \quad y>0, \alpha, \gamma, b>0,
$$

where $\alpha$ and $b$ are the shape parameters and $\gamma$ is the scale parameter.

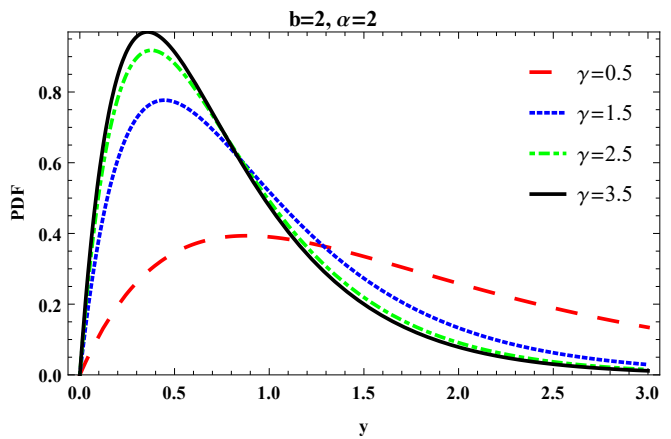

(a)

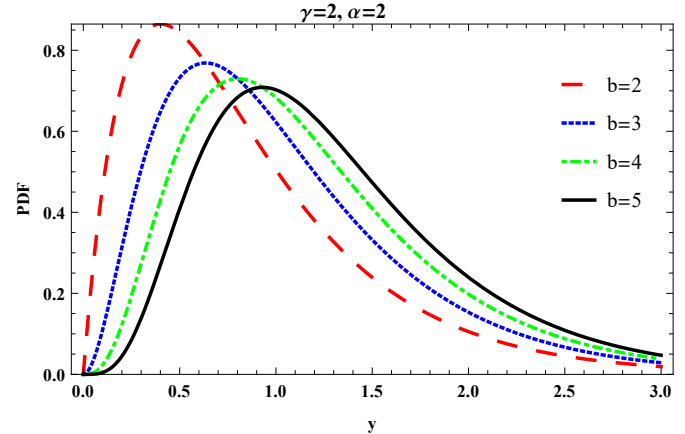

(b)

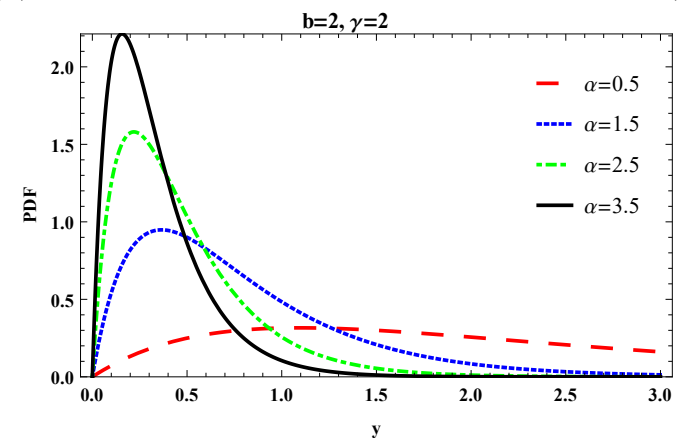

(c)

Figure 1. Plots of the pdf of TL-ETEx for different combinations of the parametric values.

Figure 1 shows the effect of different values of the parameters on the shape of the proposed distribution. For the increasing values of the shape parameter $\alpha$, the curves get more peaked and distribution becomes right-skewed. It is also observed from the Figure (a) that $\alpha$ affects the tail weight of TL-ETEx distribution. As values of $\alpha$ increase, the tail weight also increases. For increasing values of $b$, the peaks of curves start decreasing and the curves move towards the right. It is also obvious from Figure 1(c) that there appears an abrupt shift in the peak of curves and they start increasing as the values of $\gamma$ increase. The cdf and the pdf of the proposed model can be expressed in terms of the weighted sum of the exponentiated class of distributions. Using the following series representation by [23]

$$
(1-z)^{a}=\sum_{i=0}^{\infty} \frac{(-1)^{i} \Gamma(a+1)}{i ! \Gamma(a+1-i)} z^{i},
$$

the distribution function of the TL-ETEx distribution is written as

$$
F(y)=\sum_{i=0}^{\infty} \frac{(-1)^{i} \Gamma(b+1)}{i ! \Gamma(b+1-i)}\left(e^{-2 \alpha\left(1-e^{-\gamma}\right) y}\right)^{i} .
$$


Similarly, the pdf of the proposed distribution is written as

$$
f(y)=\sum_{i=0}^{\infty} \frac{(-1)^{i} 2 \Gamma(b+1)}{i ! \Gamma(b-i)} \alpha\left(1-e^{-\gamma}\right)\left(e^{-2 \alpha\left(1-e^{-\gamma}\right) y}\right)^{i+1} .
$$

Several characteristics such as moments, generating function, entropy, and order statistics can easily be computed by using the exponentiated form of the density function.

\subsection{Quantile function}

The $q^{\text {th }}$ quantile function of the TL-ETEx distribution is given as

$$
y_{q}=\frac{-\log \left(1-q^{\frac{1}{b}}\right)}{2 \alpha\left(1-e^{-\gamma}\right)}, q \in(0,1)
$$

The quantile function is used to generate the random data. The median of the proposed model can be obtained by setting $q=\frac{1}{2}$ as

$$
\text { Median }=\frac{-\log \left(1-0.5^{\frac{1}{b}}\right)}{2 \alpha\left(1-e^{-\gamma}\right)}, q \in(0,1) .
$$

\subsection{Moments}

Generally, the moments are an essential part of the distribution. They are used to find several important characteristics such as mean, variance, skewness, and kurtosis. The common method to find the raw moments or crude moments of any distribution is given as

$$
\mu_{k}^{\prime}=\int_{-\infty}^{\infty} y^{k} d F(x) .
$$

Using density function given in Equation (2.3) and assuming $w=-2 \alpha(i+1)\left(1-e^{-\gamma}\right) y$ we obtain the expression of moment of the proposed model given as

$$
\mu_{k}^{\prime}=\sum_{i=0}^{\infty} \frac{(-1)^{i} \Gamma(b+1)}{i ! \Gamma(b-i)}\left\{\frac{1}{i+1}\right\}^{k+1}\left\{\frac{1}{2 \alpha\left(1-e^{-\gamma}\right)}\right\}^{k} \Gamma(k+1) .
$$

For $k>0$, we can find different moments. The mean of TL-ETEx distribution is obtained by inserting $k=1$ expressed as

$$
\mu_{1}^{\prime}=\sum_{i=0}^{\infty} \frac{(-1)^{i} \Gamma(b+1)}{i ! \Gamma(b-i)}\left\{\frac{1}{i+1}\right\}^{2}\left\{\frac{1}{2 \alpha\left(1-e^{-\gamma}\right)}\right\} \Gamma(2) .
$$

The coefficient of variation $(C V)$, the coefficient of skewness $(C S)$, and the coefficient of kurtosis $(C K)$ of the TL-ETEx distribution are obtained as

and

$$
\begin{gathered}
C V=\sqrt{\frac{\mu_{2}}{\mu_{1}}-1}, \\
C S=\frac{\mu_{3}-3 \mu_{2} \mu_{1}+2 \mu_{1}^{3}}{\left(\mu_{2}-\mu_{1}\right)^{\frac{3}{2}}},
\end{gathered}
$$

$$
C K=\frac{\mu_{4}-4 \mu_{3} \mu_{1}+6 \mu_{2} \mu_{1}^{2}}{\left(\mu_{2}-\mu_{1}^{2}\right)^{2}} .
$$

Now, the first incomplete moment is used to derive the mean deviation, Bonferroni, and Lorenz curves. These curves have great influences in economics, reliability, demography, insurance, and medicine. The incomplete moment of a distribution is defined by

$$
\varphi_{p}(y)=\int_{0}^{t} y^{p} d F(y) .
$$


Using density function given in Equation (2.3), the incomplete moment of the TL-ETEx distribution is given as

$$
\varphi_{p}=\sum_{i=0} \frac{(-1)^{i} \Gamma(b+1)}{i ! \Gamma(b-i)}\left\{\frac{1}{i+1}\right\}^{p+1}\left\{\frac{1}{2 \alpha\left(1-e^{-\gamma}\right)}\right\}^{p} \gamma\left(p+1,2 \alpha(i+1)\left(1-e^{-\gamma}\right)\right) .
$$

The mean deviation of $Y$ about mean $\left[m_{1}=E\left(\left|Y-\mu_{1}^{\prime}\right|\right)\right]$ and mean deviation of $Y$ about median $\left[m_{2}=E(|Y-M|)\right]$ are given as $m_{1}=2 \mu_{1}^{\prime} F\left(\mu_{1}^{\prime}\right)-2 \varphi_{1}\left(\mu_{1}^{\prime}\right)$ and $m_{2}=\mu_{1}^{\prime}-2 \varphi_{1}(M)$, respectively, where $\mu_{1}^{\prime}=E(Y), M=\operatorname{Median}(Y)=Q(0.5)$, and $F\left(\mu_{1}^{\prime}\right)$ is calculated from Equation (2.4) and $\varphi_{1}(t)$ is the first incomplete moment with $p=1$. These equations for $\varphi_{1}(t)$ can be used to obtain Bonferroni and Lorenz curves for the given probability $\pi$ as $B(\pi)=\frac{\varphi_{1}(q)}{\pi \mu_{1}^{\prime}}$ and $L(\pi)=\frac{\varphi_{1}(q)}{\mu_{1}^{\prime}}$, respectively, where $\mu_{1}^{\prime}=E(Y)$ and $q=Q(\pi)$ is quantile function of $Y$ at $\pi$. The $(q, s)$ probability weighted moment (PWM) of $Y$ is defined by

$$
\rho_{q, s}=\int_{0}^{\infty} y^{q} F(y)^{s} f(y) d y .
$$

Following the above expression, the PWM of the TL-ETEx distribution is derived as

$$
\rho_{q, s}=A_{j, k} b^{*} \Gamma(q+1),
$$

where

$$
A_{j, k}=\sum_{j, k=0}^{\infty} \frac{(-1)^{j+k} \Gamma(s+1) \Gamma(b+1)}{j !, k ! \Gamma(s+1-j) \Gamma(b-k)},
$$

and

$$
b^{*}=\left\{\frac{1}{j+k+1}\right\}^{q+1}\left\{\frac{1}{2 \alpha\left(1-e^{-\gamma}\right)}\right\}^{q} .
$$

\subsection{An important distributional property}

We present an important distributional property of TL-ETEx distribution as discussed by [13].

Theorem 2.1. Let $Y$ be a non-negative and absolutely continuous random variable with $p d f$

$$
f(y)=2 b \alpha\left(1-e^{-\gamma}\right) e^{-2 \alpha\left(1-e^{-\gamma}\right) y}\left[1-e^{-2 \alpha\left(1-e^{-\gamma}\right) y}\right]^{b-1}, \quad y>0, \alpha, \gamma, b>0,
$$

and $n$-th conditional moment as

$$
E\left(Y^{n} \mid Y \leq z\right)=\frac{1}{F(z)} \int_{0}^{z} y^{n} 2 b \alpha\left(1-e^{-\gamma}\right) e^{-2 \alpha\left(1-e^{-\gamma}\right) y}\left[1-e^{-2 \alpha\left(1-e^{-\gamma}\right) y}\right]^{b-1} d y,
$$

if and only if

$$
F(z)=\left[1-e^{-2 \alpha\left(1-e^{-\gamma}\right) z}\right]^{b} .
$$

Proof of Theorem 2.1. For the necessary condition it can be easily proved that

$$
E\left(Y^{n} \mid Y \leq z\right)=\int_{0}^{z} y^{n} \frac{h(y)}{F(z)} d y .
$$

For the sufficient condition, we will proceed as

$$
\int_{0}^{z} y^{n} \frac{h(y)}{F(z)} d y=\frac{1}{\left[1-e^{-2 \alpha\left(1-e^{-\gamma}\right) z}\right]^{b}} \int_{0}^{z} y^{n} 2 b \alpha\left(1-e^{-\gamma}\right) e^{-2 \alpha\left(1-e^{-\gamma}\right) y}\left[1-e^{-2 \alpha\left(1-e^{-\gamma}\right) y}\right]^{b-1} d y .
$$

From Equation (2.5), we have

$$
\int_{0}^{z} y^{n} h(y) d y=F(z) \frac{1}{\left[1-e^{-2 \alpha\left(1-e^{-\gamma}\right) z}\right]^{b}} \int_{0}^{z} y^{n} 2 b \alpha\left(1-e^{-\gamma}\right) e^{-2 \alpha\left(1-e^{-\gamma}\right) y}\left[1-e^{-2 \alpha\left(1-e^{-\gamma}\right) y}\right]^{b-1} d y .
$$


Differentiating both sides of Equation (2.6) with respect to $z$, we get

$$
z^{n} f(z)=f(z) I_{1}+F(z) I_{2} I^{*}+F(z) \frac{1}{F(y)} I_{3} .
$$

where

$$
\begin{aligned}
& I_{1}=\frac{1}{\left[1-e^{-2 \alpha\left(1-e^{-\gamma}\right) z}\right]^{b}} \int_{0}^{z} y^{n} 2 b \alpha\left(1-e^{-\gamma}\right) e^{-2 \alpha\left(1-e^{-\gamma}\right) y}\left[1-e^{-2 \alpha\left(1-e^{-\gamma}\right) y}\right]^{b-1}, \\
& I_{2}=-\frac{1}{2 b \alpha\left(1-e^{-\gamma}\right) e^{-2 \alpha\left(1-e^{-\gamma}\right) z}\left[1-e^{-2 \alpha\left(1-e^{-\gamma}\right) z}\right]^{b-1}}, \\
& I_{3}=z^{n} 2 b \alpha\left(1-e^{-\gamma}\right) e^{-2 \alpha\left(1-e^{-\gamma}\right) z}\left[1-e^{-2 \alpha\left(1-e^{-\gamma}\right) z}\right]^{b-1}, \\
& I^{*}=\int_{0}^{z} y^{n} 2 b \alpha\left(1-e^{-\gamma}\right) e^{-2 \alpha\left(1-e^{-\gamma}\right) y}\left[1-e^{-2 \alpha\left(1-e^{-\gamma}\right) y}\right]^{b-1} .
\end{aligned}
$$

After some simplification, we have

$$
\begin{gathered}
f(z)\left\{z^{n}-W^{*}\right\}=F(z) B^{*}\left\{z^{n}-W^{*}\right\} \\
\frac{f(z)}{F(z)}=B^{*}
\end{gathered}
$$

where

$$
B^{*}=\frac{2 b \alpha\left(1-e^{-\gamma}\right) e^{-2 \alpha\left(1-e^{-\gamma}\right) z}\left[1-e^{-2 \alpha\left(1-e^{-\gamma}\right) z}\right]^{b-1}}{\left[1-e^{-2 \alpha\left(1-e^{-\gamma}\right) z}\right]^{b}} .
$$

Integrating both sides of Equation (2.7) with respect to $z$ from $z=y$ to $\infty$, we get

$$
-\ln F(y)=-\ln \left[1-e^{-2 \alpha\left(1-e^{-\gamma}\right) y}\right]^{b} .
$$

Finally, we have

$$
F(y)=\left[1-e^{-2 \alpha\left(1-e^{-\gamma}\right) y}\right]^{b}
$$

\subsection{Moment generating function and probability generating function}

The moment generating function (mgf) of $Y$ is defined as

$$
M_{t}(Y)=\int_{-\infty}^{\infty} e^{y t} d F(y)
$$

considering $e^{y t}=\sum_{r=0}^{\infty} \frac{t ! y^{r} !}{r !}$ and using pdf given in Equation (2.3), the mgf of the proposed distribution is given as

$$
M_{t}(Y)=\sum_{i, r=0}^{\infty} \frac{t !(-1)^{i} \Gamma(b+1)}{r ! i ! \Gamma(b-i)}\left\{\frac{1}{i+1}\right\}^{r+1}\left\{\frac{1}{2 \alpha\left(1-e^{-\gamma}\right)}\right\}^{r} \Gamma(r+1) .
$$

The general expression to find the probability generating function (pgf) is given as

$$
\pi_{y}=\int_{0}^{\infty} t^{y} f(y) d y .
$$

Using $t^{y}=\sum_{m=0}^{\infty} \frac{(\ln t)^{m} y^{m}}{m !}$, the pgf of the TL-ETEx distribution is given as

$$
\pi_{y}=\sum_{i, r=0}^{\infty} \frac{(\ln t)^{m}(-1)^{i} \Gamma(b+1)}{m ! i ! \Gamma(b-i)}\left\{\frac{1}{i+1}\right\}^{m+1}\left\{\frac{1}{2 \alpha\left(1-e^{-\gamma}\right)}\right\}^{m} \Gamma(m+1) .
$$




\subsection{Survival function}

The survival function which is used to explain the probability of survival of an item beyond a given time $t$ for any distribution is given by

$$
R(y)=1-F(y),
$$

so the $R(y)$ of the TL-ETEx distribution is derived as

$$
R(y)=1-\left[1-e^{-2 \alpha\left(1-e^{-\gamma}\right) y}\right]^{b} .
$$

\subsection{Hazard rate function}

The hazard rate function of any probability distribution is given as

$$
h(y)=\frac{f(y)}{1-F(y)} .
$$

The hazard rate function of TL-ETEx distribution is obtained as

$$
h(y)=\frac{2 b \alpha\left(1-e^{-\gamma}\right) e^{-2 \alpha\left(1-e^{-\gamma}\right) y}\left[1-e^{-2 \alpha\left(1-e^{-\gamma}\right) y}\right]^{b-1}}{1-\left[1-e^{-2 \alpha\left(1-e^{-\gamma}\right) y}\right]^{b}} .
$$

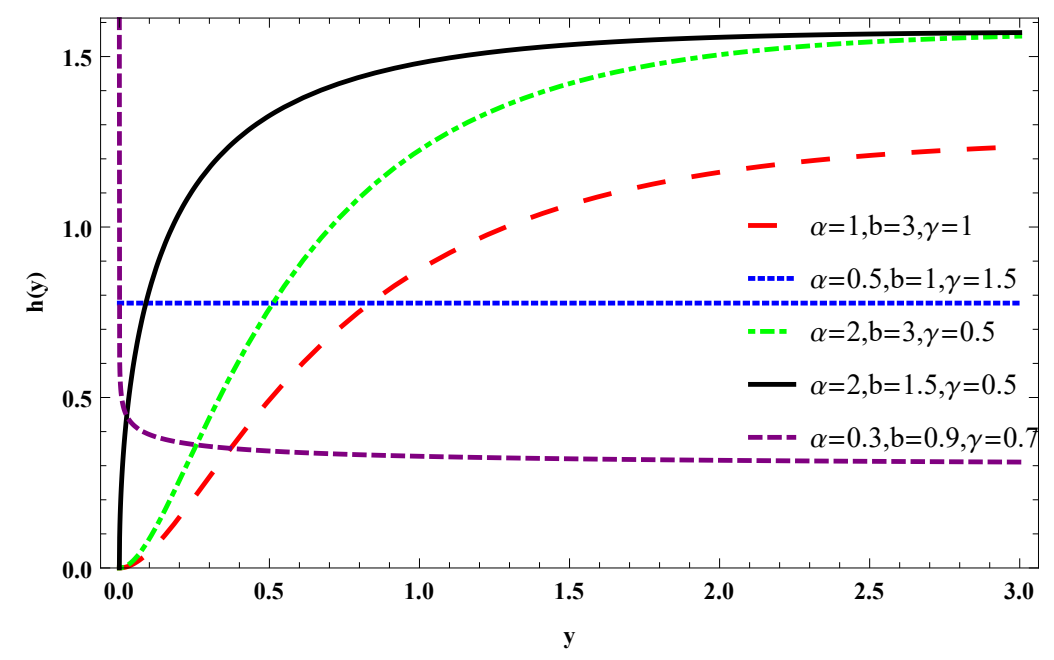

Figure 2. Hazard rate plots of TL-ETEx for different combinations of the parametric values.

The cumulative hazard rate of the proposed distribution is also given as

$$
H(y)=-\log \left\{1-\left[1-e^{-2 \alpha\left(1-e^{-\gamma}\right) y}\right]^{b}\right\} .
$$

\subsection{Residual life and reversed residual life}

The mean residual life (MRL) is the expected additional life length for a unit which survived till time $y$. The $n$th moment of the residual life is defined as

$$
\pi_{n}(t)=E\left[(Y-t)^{n} \mid Y>t\right]=\frac{1}{R(t)} \int_{t}^{\infty}(Y-t)^{n} f(y) d y, \quad n=1,2,3, \ldots
$$

After some simplifications, the above expression reduces to

$$
\pi_{n}(t)=\frac{1}{R(t)} \sum_{k, r=0}^{\infty}(-t)^{n-r} t_{k}\left(\begin{array}{l}
n \\
r
\end{array}\right) \int_{t}^{\infty} y^{r} f(y) d y .
$$


We use Equation (2.3) to solve $\int_{t}^{\infty} y^{r} f(y) d y$. After some simplifications, we obtain

$$
\pi_{n}(t)=\frac{1}{R(t)} \sum_{k, r, i, j=0}^{\infty}(-t)^{n-r} t_{k}\left(\begin{array}{l}
n \\
r
\end{array}\right) \delta_{i} \Gamma\left(p+1,2 \alpha(i+1)\left(1-e^{-\gamma}\right)\right)
$$

where

$$
\delta_{i}=\sum_{i=0} \frac{(-1)^{i} \Gamma(b+1)}{i ! \Gamma(b-i)}\left\{\frac{1}{i+1}\right\}^{p+1}\left\{\frac{1}{2 \alpha\left(1-e^{-\gamma}\right)}\right\}^{p} .
$$

We can find the mean residual life (MRL) from the above equation by replacing $n=1$. The $n$th moment of reserved residual life of any distribution is given as

$$
\kappa_{n}(t)=E\left[(t-Y)^{n} \mid Y \leq t\right]=\frac{1}{F(t)} \int_{0}^{x}(Y-t)^{n} f(y) d t, \quad n=1,2,3, \ldots
$$

The above expression can be written as

$$
\kappa_{n}(t)=\frac{1}{R(t)} \sum_{k, r=0}^{\infty}(-1)^{r}(t)^{n-r} t_{k}\left(\begin{array}{l}
n \\
r
\end{array}\right) \int_{0}^{t} y^{r} f(y) d y .
$$

The $n$th moment of the reversed residual life of the TL-ETEx distribution is given by

$$
\kappa_{n}(t)=\frac{1}{R(t)} \sum_{k, r, j, i=0}^{\infty}(-1)^{r}(t)^{n-r} t_{k}\left(\begin{array}{l}
n \\
r
\end{array}\right) \delta_{i} \gamma\left(p+1,2 \alpha(i+1)\left(1-e^{-\gamma}\right)\right) .
$$

\section{Entropy and extropy}

In this section, we compute some measures of entropy and extropy. Entropy measures the average amount of information provided by the findings of a random experiment whereas extropy is the complementary measure of entropy.

\subsection{Renyi entropy}

A generalized Renyi entropy of order $\theta$ introduced by [25] is given as

$$
H_{\theta}(Y)=\frac{1}{1-\theta} \log \int_{0}^{\infty}(f(y))^{\theta} d y .
$$

Using $f(y)$ given by Equation (2.2), the above expression for the TL-ETEx distribution is obtained as

$$
H_{\theta}(Y)=\frac{1}{1-\theta} \log \int_{0}^{\infty} b^{\theta}\left(2 \alpha\left(1-e^{-\gamma}\right)\right)^{\theta}\left(e^{-2 \alpha\left(1-e^{-\gamma}\right) y}\right)^{\theta}\left[1-e^{-2 \alpha\left(1-e^{-\gamma}\right) y}\right]^{\theta(b-1)} d y .
$$

Using the series representation given in [23], the above expression is reduced as

$$
H_{\theta}(Y)=\frac{1}{1-\theta} \log \int_{0}^{\infty} M_{j} b^{\theta}\left(2 \alpha\left(1-e^{-\gamma}\right)\right)^{\theta}\left(e^{-2 \alpha\left(1-e^{-\gamma}\right) y}\right)^{\theta+j} d y
$$

where

$$
M_{j}=\sum_{j=0}^{\infty}(-1)^{j} \frac{\Gamma(\theta(b-1)+1)}{j ! \Gamma(\theta(b-1)+1-j)} .
$$

Transforming $z=2(\theta+j) \alpha\left(1-e^{-\gamma}\right) y$, and performing some calculation the Renyi entropy of TL-ETEx distribution is given as

$$
H_{\theta}(Y)=\frac{1}{1-\theta} \log \left(M_{j} b^{\theta}\left(2 \alpha\left(1-e^{-\gamma}\right)\right)^{\theta-1}\right) .
$$




\subsection{Tsallis entropy}

A generalized Tsallis entropy of order $\theta$ introduced by[27] is given as

$$
S_{\theta}(Y)=\frac{1}{1-\theta}\left(1-\int_{0}^{\infty}(f(y))^{\theta} d y\right) .
$$

Using Equation (2.2) and simplifying the above expression, the Tsallis entropy of the proposed distribution is obtained as

$$
S_{\theta}(Y)=\frac{1}{1-\theta}\left(1-\left(M_{j} b^{\theta}\left(2 \alpha\left(1-e^{-\gamma}\right)\right)^{\theta-1}\right)\right)
$$

Both entropies have strong relationship with Shannon entropy. For $\theta \rightarrow 0$, both Renyi and Tsallis entropies approache to Shannon entropy.

Tsallis entropy plays a key role in several areas such as physics, chemistry, biology, medicine, and economics. Different applications of Tsallis entropy are discussed by [5]. The major difference between Renyi and Tsallis entropies is that the Tsallis entropy is nonextensive and nonlogarithmic whereas Renyi is extensive. The relationship between the both entropies can be expressed as

$$
H_{\theta}(Y)=\frac{1}{1-\theta} \log \left[1-(\theta-1) S_{\theta(Y)}\right] .
$$

\subsection{Cumulative residual Tsallis entropy}

The Cumulative Residual Tsallis Entropy (CRTE) of order $\theta$ defined by [26] is given as

$$
\eta_{\theta}(Y)=\frac{1}{1-\theta}\left(1-\int_{0}^{\infty}(R(y))^{\theta} d y\right),
$$

where $R(y)=\bar{F}(y)=1-F(y)$. Considering Equation (2.8), the above expression is written as

$$
\eta_{\theta}(Y)=\frac{1}{1-\theta}\left(1-\int_{0}^{\infty}\left(1-\left[1-e^{-2 \alpha\left(1-e^{-\gamma}\right) y}\right]^{b}\right)^{\theta} d y\right) .
$$

Using the series representation given by [23], the above expression becomes

$$
\eta_{\theta}(Y)=\frac{1}{1-\theta}\left(1-\int_{0}^{\infty} L_{j, k}^{*} e^{-2 \alpha\left(1-e^{-\gamma}\right) y k} d y\right)
$$

where

$$
L_{j, k}^{*}=\sum_{j, k=0}^{\infty}(-1)^{j+k} \frac{\Gamma(\theta+1)(b j+1)}{j ! k ! \Gamma(\theta+1-j)(b j+1-k)} .
$$

After solving Equation (3.1), the CRTE of TL-ETEx distribution is given as

$$
\eta_{\theta}(Y)=\frac{1}{1-\theta}\left(1-L_{j, k}^{*} \frac{1}{2 k \alpha\left(1-e^{-\gamma}\right)}\right) .
$$

\subsection{Dynamic cumulative residual Tsallis entropy}

For a random variable $Y_{t}$ with survival function $R(y)$, the dynamic cumulative residual Tsallis entropy (DCRTE) of order $\theta$ is defined as

$$
\eta_{\theta}(Y ; t)=\frac{1}{1-\theta}\left(1-\frac{1}{R(t)} \int_{t}^{\infty}(R(y))^{\theta} d y\right) .
$$

Considering Equation (2.8), the DCRTE of TL-ETEx distribution is given as

$$
\eta_{\theta}(Y ; t)=\frac{1}{1-\theta}\left(1-\frac{1}{R(t)} L_{j, k}^{*} \frac{e^{\gamma+2 \alpha\left(e^{-\gamma}-1\right) k t}}{2 \alpha\left(e^{\gamma}-1\right) k}\right) .
$$




\subsection{Residual extropy}

The residual extropy proposed by [24] as the measure of residual uncertainty of a random variable is

$$
J(Y ; t)=-\frac{1}{2 R(t)^{2}} \int_{t}^{+\infty} f(y)^{2} d y .
$$

The residual extropy of TL-ETEx distribution is obtained by using Equation (2.2) and is given as

$$
J(Y)=-T_{j} b^{2} \alpha\left(1-e^{-\gamma}\right) e^{-t}
$$

\subsection{Cumulative residual extropy}

A new measure of extropy called cumulative residual extropy (CRE) introduced by [11] is

$$
\xi J(X)=-\frac{1}{2} \int_{0}^{\infty} R(y)^{2} d y .
$$

Using Equation (2.8), the CRE of the proposed distribution is obtained as

where

$$
\xi J(X)=-t_{j, k}^{*} \frac{1}{4 \alpha\left(1-e^{-\gamma}\right) k},
$$

$$
t_{j, k}^{*}=\sum_{k=0}^{\infty} \sum_{j=0}^{3}(-1)^{j+k} \frac{\Gamma(b j+1)(3)}{j ! k ! \Gamma(b j+1-k)(3-j)} .
$$

\section{Inference}

In this section, we discuss the estimation of the unknown parameters of the proposed distribution by employing the method of maximum likelihood estimation. Let a random sample, $y_{1}, y_{2}, \ldots, y_{n}$ of size $n$ is drawn from the TL-ETEx distribution then the maximum likelihood estimates (MLEs) of its parameters are obtained by using the following likelihood $(L)$ function

$$
L=\prod_{i=1}^{n} 2 b \alpha\left(1-e^{-\gamma}\right) e^{-2 \alpha\left(1-e^{-\gamma}\right) y_{i}}\left[1-e^{-2 \alpha\left(1-e^{-\gamma}\right) y_{i}}\right]^{b-1},
$$

and log-likelihood (l) function

$$
\begin{aligned}
l= & n \log 2+n \log b+n \log \alpha+n \log \left(1-e^{-\gamma}\right)-2 \alpha\left(1-e^{-\gamma}\right) \sum_{i=1}^{n} y_{i} \\
& +(b-1) \sum_{i=1}^{n} \log \left[1-e^{-2 \alpha\left(1-e^{-\gamma}\right) y_{i}}\right] .
\end{aligned}
$$

Taking the partial derivatives of Equation (4.1) with respect to $\alpha, \gamma$, and $b$ and setting resulting equations equal to zero, we obtain a system of three equations in three unknowns parameters are given as

$$
\begin{gathered}
\frac{\partial L}{\partial b}=\frac{n}{b}+\sum_{i=1}^{n} \log \left(1-e^{-2 \alpha\left(1-e^{-\gamma}\right) y_{i}}\right)=0 \\
\frac{\partial L}{\partial \alpha}=\frac{n}{\alpha}-2\left(1-e^{-\gamma}\right) \sum_{i=1}^{n} y_{i}+(b-1) 2\left(1-e^{-\gamma}\right) \sum_{i=1}^{n} \frac{y_{i} e^{-2 \alpha\left(1-e^{-\gamma}\right) y_{i}}}{1-e^{-2 \alpha\left(1-e^{-\gamma}\right) y_{i}}}=0
\end{gathered}
$$




$$
\frac{\partial L}{\partial \gamma}=\frac{n e^{-\gamma}}{1-e^{-\gamma}}-2 \alpha e^{-\gamma} \sum_{i=1}^{n} y_{i}+(b-1) 2 \alpha e^{-\gamma} \sum_{i=1}^{n} \frac{y_{i} e^{-2 \alpha\left(1-e^{-\gamma}\right) y_{i}}}{1-e^{-2 \alpha\left(1-e^{-\gamma}\right) y_{i}}}=0 .
$$

It is important to point out here that the resulting analytical solution of the system of non linear Equations (4.2), (4.3), and (4.4) are unknown. So, the estimates of $\hat{b}, \hat{\alpha}$, and $\hat{\gamma}$ are obtained by solving the system of these equations using some numerical methods.

The Fisher Information matrix $I(b, \alpha, \gamma)$, assessed by taking the minus expectations of the second partial derivatives of Equations (4.2), (4.3), and (4.4), is expressed as

$$
\begin{gathered}
\left(\begin{array}{c}
\hat{b} \\
\hat{\alpha} \\
\hat{\gamma}
\end{array}\right) \sim N\left(\left(\begin{array}{l}
b \\
\alpha \\
\gamma
\end{array}\right)\left(\begin{array}{lll}
\hat{J}_{b b} & \hat{J}_{b \alpha} & \hat{J}_{b \gamma} \\
& \hat{J}_{\alpha \alpha} & \hat{J}_{\alpha \gamma} \\
& & \hat{J}_{\gamma \gamma}
\end{array}\right)\right), \\
\frac{1}{J}=-E\left(\begin{array}{ccc}
J_{b b} & J_{b \alpha} & J_{b \gamma} \\
& J_{\alpha \alpha} & J_{\alpha \gamma} \\
& & J_{\gamma \gamma}
\end{array}\right) .
\end{gathered}
$$

The entries of the Fisher Information matrix are given as

$$
\begin{aligned}
& \hat{J}_{b b}=-\frac{n}{b^{2}}, \\
& \hat{J}_{b \alpha}=\sum_{i=1}^{n} \frac{2(w) y_{i} e^{-2 \alpha(w) y_{i}}}{1-e^{-2 \alpha(w) y_{i}}} \\
& \hat{J}_{b \gamma}=\sum_{i=1}^{n} \frac{2 \alpha e^{-\gamma} y_{i} e^{-2 \alpha(w) y_{i}}}{1-e^{-2 \alpha(w) y_{i}}} \\
& \hat{J}_{\alpha \alpha}=-\frac{n}{\alpha^{2}}-(b-1) \sum_{i=1}^{n} \frac{4(w)^{2} y_{i}^{2} e^{-4 \alpha(w) y_{i}}}{\left(1-e^{-2 \alpha(w) y_{i}}\right)^{2}}-\sum_{i=1}^{n} \frac{4(w)^{2} y_{i}^{2} e^{-2 \alpha(w) y_{i}}}{1-e^{-2 \alpha(w) y_{i}}}, \\
& \hat{J}_{\alpha \gamma}=-2 e^{-\gamma} \sum_{i=1}^{n} y_{i}-(1-b) \sum_{i=1}^{n}\left(\frac{4 \alpha e^{-\gamma}(w) y_{i}^{2} e^{-4 \alpha(w) y_{i}}}{\left(1-e^{-2 \alpha(w) y_{i}}\right)^{2}}\right) \\
& -(b-1) \sum_{i=1}^{n}\left(\frac{4 \alpha e^{-\gamma}(w) y_{i}^{2} e^{-2 \alpha(w) y_{i}}}{1-e^{-2 \alpha(w) y_{i}}}+\frac{2 e^{-\gamma} y_{i} e^{-2 \alpha(w) y_{i}}}{1-e^{-2 \alpha(w) y_{i}}}\right), \\
& \hat{J}_{\gamma \gamma}=\frac{n e^{-\gamma}}{(w)^{2}}+2 \alpha e^{-\gamma}\left(\sum_{i=1}^{n} y_{i}\right)-(b-1) \sum_{i=1}^{n}\left(-\frac{4 \alpha^{2} e^{-2 \gamma} y_{i}^{2} e^{-4 \alpha(w) y_{i}}}{\left(1-e^{\left.-2 \alpha(w) y_{i}\right)^{2}}\right.}\right) \\
& -(b-1) \sum_{i=1}^{n}\left(\frac{4 \alpha^{2} e^{-2 \gamma} y_{i}^{2} e^{-2 \alpha(w) y_{i}}}{1-e^{-2 \alpha(w) y_{i}}}+\frac{2 \alpha e^{-\gamma} y_{i} e^{-2 \alpha(w) y_{i}}}{1-e^{-2 \alpha(w) y_{i}}}\right),
\end{aligned}
$$

where $1-e^{-\gamma}=w$.

\section{Simulation}

In this section, we perform a simulation study to evaluate the performance of MLEs of the unknown parameters of the TL-ETEx distribution. We generate $N=10000$ simulations of the TL-ETEx distribution for different sample sizes $n=50,250,500,1000$ using the following equation

$$
y_{q}=\frac{-\log \left(1-q^{\frac{1}{b}}\right)}{2 \alpha\left(1-e^{-\gamma}\right)}, q \in(0,1),
$$

where $U$ is a random variable that follows the uniform distribution on the interval $[0,1]$. Table 1 lists the average estimated (AE)values of the parameters and their mean square errors (MSEs). 
Table 1. Average estimated values of the parameters and their mean square errors for several combinations of the parameters of TL-ETEx distribution.

\begin{tabular}{|c|c|c|c|c|c|c|c|c|c|}
\hline \multirow[t]{2}{*}{$\mathrm{n}$} & \multicolumn{3}{|c|}{ True values } & \multicolumn{3}{|c|}{$\mathrm{AE}$} & \multicolumn{3}{|c|}{ MSEs } \\
\hline & $\alpha$ & $b$ & $\gamma$ & $\hat{\alpha}$ & $\hat{b}$ & $\hat{\gamma}$ & $\hat{\alpha}$ & $\hat{b}$ & $\hat{\gamma}$ \\
\hline \multirow{5}{*}{50} & 0.5 & 0.5 & 1 & 0.5177 & 0.5313 & 1.0354 & 0.0368 & 0.0470 & 0.0791 \\
\hline & 1.5 & 1.5 & 0.5 & 1.5193 & 1.5298 & 0.5414 & 0.0451 & 0.0741 & 0.0950 \\
\hline & 2 & 1.5 & 2.5 & 2.0216 & 1.5222 & 2.5311 & 0.0535 & 0.0307 & 0.0454 \\
\hline & 2.5 & 2 & 2.5 & 2.5181 & 2.0343 & 2.5311 & 0.0247 & 0.0849 & 0.0547 \\
\hline & 2.5 & 0.5 & 2 & 2.5223 & 0.5271 & 2.0433 & 0.0563 & 0.0483 & 0.1024 \\
\hline \multirow{5}{*}{250} & 0.5 & 0.5 & 1 & 0.5045 & 0.5069 & 1.0096 & 0.0160 & 0.01901 & 0.0338 \\
\hline & 1.5 & 1.5 & 0.5 & 1.5051 & 1.5081 & 0.5103 & 0.0191 & 0.0258 & 0.0374 \\
\hline & 2 & 1.5 & 2.5 & 2.0036 & 1.5065 & 2.5098 & 0.0069 & 0.0165 & 0.0443 \\
\hline & 2.5 & 2 & 2.5 & 2.5054 & 2.0077 & 2.5099 & 0.0248 & 0.0297 & 0.0375 \\
\hline & 2.5 & 0.5 & 2 & 2.5042 & 0.5078 & 2.0120 & 0.0091 & 0.0296 & 0.0548 \\
\hline \multirow{5}{*}{500} & 0.5 & 0.5 & 1 & 0.5030 & 0.5037 & 1.0059 & 0.0159 & 0.0165 & 0.0287 \\
\hline & 1.5 & 1.5 & 0.5 & 1.5026 & 1.5039 & 0.5059 & 0.0093 & 0.0157 & 0.0313 \\
\hline & 2 & 1.5 & 2.5 & 2.0022 & 1.5036 & 2.5053 & 0.0060 & 0.0147 & 0.0199 \\
\hline & 2.5 & 2 & 2.5 & 2.5032 & 2.0042 & 2.5055 & 0.0178 & 0.0232 & 0.0202 \\
\hline & 2.5 & 0.5 & 2 & 2.5025 & 0.5044 & 2.0063 & 0.0086 & 0.0196 & 0.0341 \\
\hline \multirow{5}{*}{1000} & 0.5 & 0.5 & 1 & 0.5013 & 0.5016 & 1.0034 & 0.0048 & 0.0053 & 0.0235 \\
\hline & 1.5 & 1.5 & 0.5 & 1.5014 & 1.5023 & 0.5028 & 0.0054 & 0.0153 & 0.0149 \\
\hline & 2 & 1.5 & 2.5 & 2.0015 & 1.5022 & 2.5033 & 0.0086 & 0.0103 & 0.0263 \\
\hline & 2.5 & 2 & 2.5 & 2.5017 & 2.0023 & 2.5024 & 0.012 & 0.0159 & 0.0096 \\
\hline & 2.5 & 0.5 & 2 & 2.5014 & 0.5024 & 2.0026 & 0.0066 & 0.0199 & 0.0094 \\
\hline
\end{tabular}

Table 1 indicates that the estimates are quite stable and are close to the true values for different sample sizes. It is observed that in general the average estimated values of the parameters become close to the true values while the MSEs decrease as $n$ increases.

\section{Application}

In this section, we study the applicability of the TL-ETEx distribution by means of three real data sets from the fields of hydrology, reliability, and environmental sciences. We compare the results of the proposed distribution with some existing distributions. We consider-2 2 ( maximized log-likelihood), $A I C$ (Akaike information criterion), and BIC (Bayesian Information criterion) as the goodness of fit measures. The compared models are listed below in Table 2.

Table 2. Fitted distributions and their abbreviations.

\begin{tabular}{llc}
\hline Model & Abbreviations & Referrence \\
\hline Topp Leone Erlang Truncated Exponential & TL-ETEx & Proposed \\
Erlang Truncated Exponential & ETEx & {$[7]$} \\
Exponential & Ex & {$[4]$} \\
Marshall-Oklin Erlang Truncated Exponential & MO-ETEx & {$[21]$} \\
Inverse Weibull & IEx & {$[12]$} \\
Topp Leone Inverse Weibull & TL-IW & {$[1]$} \\
\hline
\end{tabular}

The first data set represents the level of mercury in 34 albacores caught in the Eastern Mediterranean used by [15]. The second data set consists of thirty successive values of March precipitation (in inches) in Minneapolis/St Paul by [10]. The third data set represents the waiting times (in minutes) before the service of 100 Bank customers which was examined and analyzed by [8].

The estimated parameters of the fitted distributions, $-2 \ell, A I C$ and $B I C$ are listed in Tables [3-5] for the selected three data sets, respectively. The values in these tables reveal 
that the TL-ETEx distribution provides better fits than other fitted models. The fitted density, empirical cdf, and total time on test (TTT) plots of the TL-ETEx distribution are displayed in Figures 3-5 for the three data sets, respectively. These figures show that the TL-ETEx distribution fits all data sets adequately. The TTT plot shows that all data sets have a monotonically increasing hazard rate function.

Table 3. Estimated parameters, $-2 \ell, A I C$, and $B I C$ for the fitted distributions for data set I.

\begin{tabular}{lcccccc}
\hline \multirow{2}{*}{ Model } & \multicolumn{3}{c}{ Parameters } & $-2 \ell$ & AIC & BIC \\
\cline { 2 - 4 } & $\alpha$ & $\beta$ & $\gamma$ & & & \\
\hline TL-ETEx & 1.38714 & 17.2665 & 2.4083 & 20.2687 & 46.53747 & 51.1165 \\
ETEx & 1.1229 & 1.0662 & & 44.40812 & 92.8162 & 95.8689 \\
Ex & 0.7363 & & & 44.40812 & 90.8162 & 92.3426 \\
MO-ETEx & 155.9432 & 4.0686 & 2.6041 & 20.7923 & 47.5847 & 52.1637 \\
IW & 1.2301 & & & 62.2802 & 126.5605 & 128.0869 \\
TL-IW & 2.3288 & 3.9152 & 0.4059 & 22.1965 & 50.3930 & 54.9721 \\
\hline
\end{tabular}

Table 4. Estimated parameters, $-2 \ell, A I C$, and $B I C$ for fitted distributions for data set II.

\begin{tabular}{lcccccc}
\hline \multirow{2}{*}{ Model } & \multicolumn{3}{c}{ Parameters } & $-2 \ell$ & AIC & BIC \\
\cline { 2 - 4 } & $\alpha$ & $\beta$ & $\gamma$ & & & \\
\hline TL-ETEx & 0.7157728 & 3.46107 & 1.6886 & 38.0942 & 82.1885 & 86.3921 \\
ETEx & 0.9596 & 0.9732 & & 45.4744 & 94.9488 & 97.7512 \\
Ex & 0.7362973 & & & 44.40812 & 90.81625 & 92.34261 \\
MO-ETEx & 9.97431 & 4.8276 & 0.3885 & 39.377 & 84.754 & 88.9576 \\
IW & 0.8014 & & & 69.0502 & 140.1003 & 141.5015 \\
TL-IW & 1.2951 & 3.5629 & 0.3767 & 40.1633 & 86.3266 & 90.5302 \\
\hline
\end{tabular}

Table 5. Estimated parameters, $-2 \ell, A I C$, and $B I C$ for fitted distributions for data set III.

\begin{tabular}{lcccccc}
\hline Model & \multicolumn{3}{c}{ Parameters } & $-2 \ell$ & AIC & BIC \\
\cline { 2 - 4 } & $\alpha$ & $\beta$ & $\gamma$ & & & \\
\hline TL-ETEx & 0.2508 & 2.1826 & 0.3814 & 317.0953 & 640.1906 & 648.0061 \\
ETEx & 0.2043 & 0.6842 & & 329.0209 & 662.0418 & 667.2521 \\
Ex & 0.1012 & & & 329.0209 & 660.0418 & 662.6469 \\
MO-ETEx & 4.1164 & 1.3268 & 0.15662 & 320.712 & 647.4241 & 655.2396 \\
IW & 0.1922 & & & 554.9761 & 1111.952 & 1114.557 \\
TL-IW & 0.9567 & 12.5285 & 0.5246 & 327.1056 & 660.2112 & 668.0267 \\
\hline
\end{tabular}

\section{Conclusion}

We propose a new lifetime distribution called Topp Leone Erlang truncated exponential (TL-ETEx) distribution by generalizing the Erlang truncated exponential distribution using Topp Leone family of distributions. Several important characteristics of the proposed distribution are derived and discussed. The moments of TL-ETEx distribution help to study its behavior while the graphical representation describes the impact of different parametric combinations on the peak and the skewness of the proposed distribution. It is observed from the reliability analysis that the TL-ETEx distribution has increasing, decreasing and constant failure rates which indicates its ability to handle the versatile situations. Various types of entropies and extropies are derived which help to find the average amount of uncertainty in the information provided by the random trials. The 


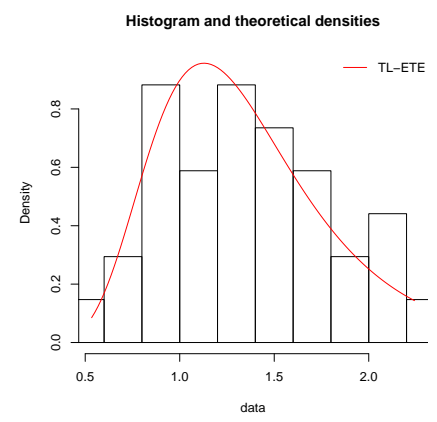

Data set I

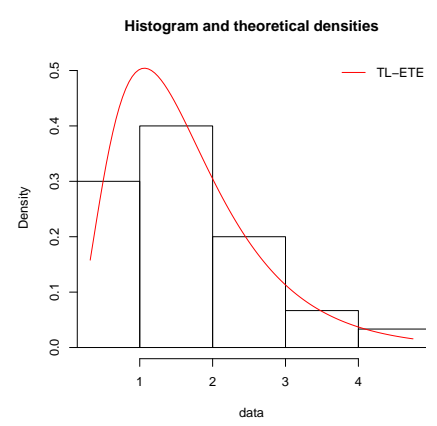

Data set II

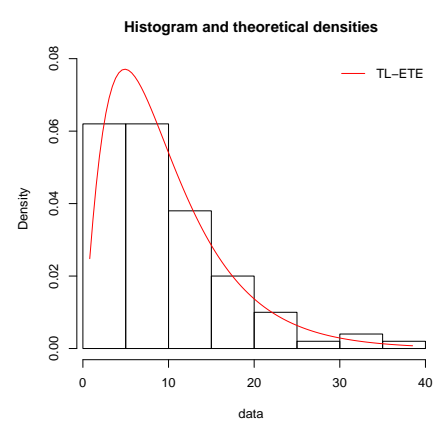

Data set III

Figure 3. Fitted density of the TL-ETEx distribution for three data sets.

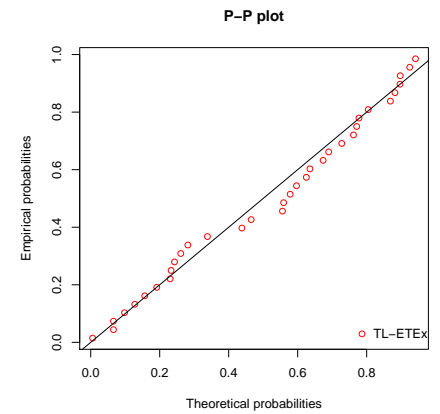

Data set I

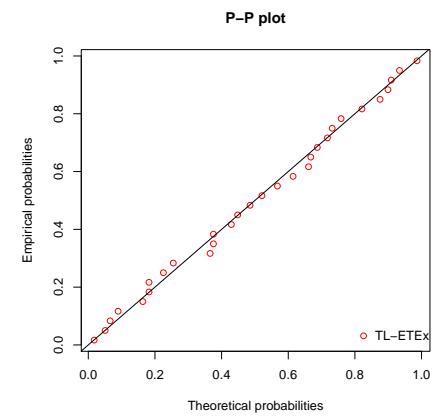

Data set II

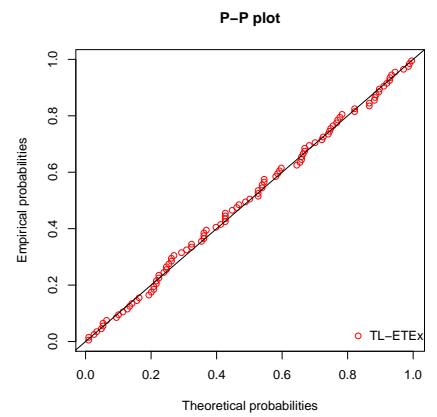

Data set III

Figure 4. Probability plots of the TL-ETEx distribution for three data sets.

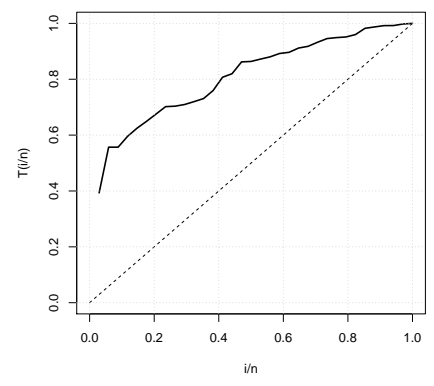

Data set I

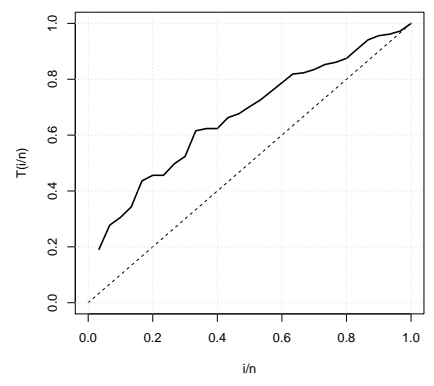

Data set II

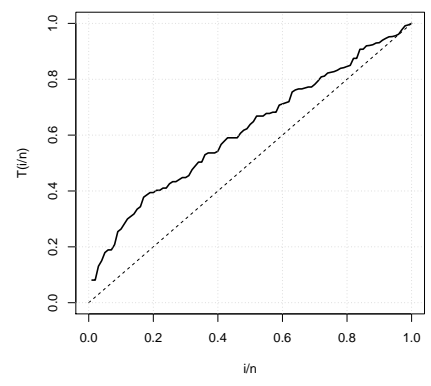

Data set III

Figure 5. TTT plots of the TL-ETEx distribution for three data sets.

findings of the simulation study reveal that the estimated values of the parameters are close to their true values which establish their stability. It is also noticed that the estimated values become closer to the true values where as the biases and standard errors decrease as the samples sizes increase. Three real data sets from hydrology, reliability, and environmental sciences are modeled by using the TL-ETEx distribution that endorse its vast application. 
Acknowledgment. The authors are thankful to the editor and the reviewers for their valuable comments and suggestions which certainly helped to improve the paper.

\section{References}

[1] S. Abbas, S.A. Taqi, F. Mustafa, M. Murtaza and M.Q. Shahbaz, Topp-Leone inverse Weibull distribution: Theory and application, Eur. J. Appl. Math. 10 (5), 1005-1022, 2017.

[2] S. Abu-Youssef, B. Mohammed and M. Sief, An extended exponentiated exponential distribution and its properties, Int. J. Comput. Appl. 121 (5), 1-6, 2015.

[3] A.Z. Afify, M. Zayed, and M. Ahsanullah, The extended exponential distribution and its applications, J. Stat. Theory Appl. 17 (2), 213-229, 2018.

[4] A. Al-Shomrani, O Arif, A. Shawky, S. Hanif and M.Q. Shahbaz, Topp-Leone family of distributions: Some properties and application, Pak. J. Stat. Oper. Res. 12 (3), 443-451, 2016.

[5] J. Cartwright, Roll over, Boltzmann, Physics World 27 (05), 31-35, 2014

[6] S.T. Dara and M. Ahmad, Recent Advances in Moment Distribution and their Hazard Rates, LAP LAMBERT Academic Publishing, 2012.

[7] A.R. El-Alosey, Random sum of new type of mixture of distribution, International Journal of Statistics and Systems 2 (1), 49-57, 2007.

[8] M. Ghitany, D.K. Al-Mutairi, N. Balakrishnan and L. Al-Enezi, Power Lindley distribution and associated inference, Comput. Statist. Data Anal. 64, 20-33, 2013.

[9] R.D. Gupta and D. Kundu, Theory \& methods: Generalized exponential distributions, Aust. N. Z. J. Stat. 41 (2), 173-188, 1999.

[10] D. Hinkley, On quick choice of power transformation J. R. Stat. Soc. Ser. C. Appl. Stat. 26 (1), 67-69, 1977.

[11] S. Jahanshahi, H. Zarei and A. Khammar, On cumulative residual extropy, Probab. Engrg. Inform. Sci. 34 (4), 605-625, 2020.

[12] A. Keller, A. Kamath and U. Perera, Reliability analysis of CNC machine tools, Reliab. Eng. 3 (6), 449-473, 1982.

[13] M. Mohsin, M. Ahmad, S. Shahbaz and M.Q. Shahbaz, Concomitant of lower record for bivariate pseudo inverse rayleigh distribution, Sci.Int. (Lahore) 21 (1), 21-23, 2009.

[14] M. Mohsin, S. Shahbaz and M.Q. Shahbaz, A characterization of Erlang-truncated exponential distribution in record values and its use in mean residual life, Pak. J. Stat. Oper. Res. 6 (2), 143-148, 2010.

[15] S. Mol, O. Ozden and S. Karakulak, Levels of selected metals in albacore (Thunnus alalunga, Bonnaterre, 1788) from the Eastern Mediterranean, J. Aquat. Food Prod. Technol. 21 (2), 111-117, 2012.

[16] S. Nadarajah and S. Kotz, Moments of some J-shaped distributions, J. Appl. Stat. 30 (3), 311-317, 2003.

[17] S. Nadarajahand and S. Kotz, The beta exponential distribution, Reliab. Eng. Syst. Saf. 91 (6), 689-697, 2006.

[18] S. Nasiru, M. Atem and K. Nantomah, Poisson exponentiated Erlang-truncated exponential distribution, J. Stat. Appl. Probab. 7 (2), 245-261, 2018.

[19] S. Nasiru, A. Luguterah and M.M. Iddrisu, Generalized Erlang-truncated exponential distribution, Advances and Applications in Statistics 48 (4), 273-301, 2016.

[20] I.E. Okorie, C.A. Akpanta and J. Ohakwe, Transmuted Erlang-truncated exponential distribution, Econ. Qual. Control 31 (2), 71-84, 2016.

[21] I.E. Okorie, A. Akpanta and J. Ohakwe, Marshall-Olkin generalized Erlang-truncated exponential distribution: Properties and applications, Cogent Math. 4 (1), 1285093, 2017. 
[22] I.E. Okorie, A. Akpanta, J. Ohakwe and D. Chikezie, The extended Erlang-truncated exponential distribution: Properties and application to rainfall data, Heliyon 3 (6), e00296, 2017.

[23] A. Prudnikov, Y.A. Brychkov and O.I. Marichev, Integrals and Series, More Special Functions, CRC Press, 1986.

[24] G. Qiu and K. Jia, The residual extropy of order statistics, Statist. Probab. Lett. 133, $15-22,2018$.

[25] A. Renyi, On measures of entropy and information, in: Proceedings of the Fourth Berkeley Symposium on Mathematical Statistics and Probability, Contributions to the Theory of Statistics, The Regents of the University of California, 1961.

[26] M.M. Sati and N. Gupta, Some characterization results on dynamic cumulative residual tsallis entropy, J. Probab. Stat., Doi:10.1155/2015/694203, 2015.

[27] C. Tsallis, Possible generalization of Boltzmann-Gibbs statistics, J. Stat. Phys. 52 (1-2), 479-487, 1988. 